\title{
Photovoltaic Greenhouses: Comparison of Optical and Thermal Behaviour for Energy Savings
}

\author{
Maurizio Carlini, Tommaso Honorati, and Sonia Castellucci
}

CIRDER, Tuscia University, San Camillo de Lellis Street, 01100 Viterbo, Italy

Correspondence should be addressed to Tommaso Honorati, honorati@unitus.it

Received 29 September 2011; Accepted 15 November 2011

Academic Editor: Carlo Cattani

Copyright (C) 2012 Maurizio Carlini et al. This is an open access article distributed under the Creative Commons Attribution License, which permits unrestricted use, distribution, and reproduction in any medium, provided the original work is properly cited.

The production of energy from renewable sources, the diversification of the productive activities, and the development of photovoltaic technology and integrated systems have led to the development of solar greenhouses. The interest of the developers and designers is now to seek new approaches to combine the electricity and food production optimally. The interaction of factors as outside local climate, exposure, slope, soil, altitude, wind conditions, structural materials, or cultivated plant species, influences greatly the energy balance. This paper illustrates the comparison of optical and thermal behavior of a solar greenhouse and a similar glass greenhouse, devoted to the production of soil-less tomatoes in three different Italian areas, with computational aspects and methods of the TRNSYS simulation. Values of climatic parameters are obtained as a responce for the feasibility of the cultivation under PV modules. The results show energy savings both for heating and cooling due to PV panels, adding a new reason for the realization of these systems.

\section{Introduction}

The development of solar greenhouses in Italy is due to the current trend to diversify agricultural production, energy efficiency, and farmer's specialization. In a greenhouse there are significant interactions between different factors of production, including the different structural type and climate along the Italian territory. For the various issues of this agroecosystem, a systematic and multidisciplinary approach is pursued in which the use of different available technologies can heal the conflict that still exists between food production, energy demand, environmental protection, and economic policy. In this context, an innovative strategy to reduce the impact of protected cultivation on the environment influence is one that aims to transform the emissions from the agricultural system "open" to a kind of "closed," essentially based on the reuse or reduction of waste material, and on the computerized monitoring of pests on the "soil-less" crop and the recycling of the nutrient solution. 
Approximately $20-30 \%$ of the Italian greenhouses are equipped with heating and cooling systems. It was calculated that only the direct consumption of energy for the air conditioning goes whirling on the order of 140,000 TOE (tons of oil equivalent), approximately $90-95 \%$ of global energy demand for production.

However, the interaction of factors that influence the design and use of the greenhouse (outside local climate, exposure, slope, soil, altitude, wind conditions, type of glass and structural material used, cultivated plant species, etc.) influences greatly the energy balance [1]. Therefore, the focus of research and experimentation has been devoted to the study of variables in order to optimize the conditioner system [2].

To simulate a greenhouse has been proposed several studies to obtain values forecasts or simulations of influential variables for protected crops, such as ventilation, the water temperature for hydroponic systems, the control of $\mathrm{CO}_{2}$ for carbon fertilization, the moisture budget, climate control, and heat exchange. Recently, the thermal behavior of the greenhouses was studied using dynamic thermal simulation tool TRNSYS 15.1.

Due to the actual strain of researching optimal solutions for the use of resources, it is important to create a model that includes all variable influential on greenhouse microclimate [3]. In this study, a simulation project with TRNSYS 17 software has been created for analizing the optical and thermal behavior of a soil-less tomato crop. Values of climatic parameters are obtained comparing a glass greenhouse to a photovoltaic greenhouse in three different locations in Italy as a responce for the feasibility of the cultivation under PV modules.

\section{Materials and Methods}

The study has been developed taking an existing greenhouse as reference and drawing it through Google SketchUp software. Then it was repeated for an analogue greenhouse with PV modules partially covered. Both the structures has been used for creating two different simulation projects considering soil-less tomato climate requirements. Projects relate to our work yet described [1], modified with new methods for solar radiation carried out with TRNSYS 17. Three different locations are considered, Turin, Rome, and Ragusa, representative, respectively for the North, Centre, and South Italy.

The choice of three different locations is due to the fact that in Italy the tomato is grown in all three areas where climatic conditions are significantly different. Soil-less tomato growing is a technique that can be carried out in all three environments and thus represents an essential link between farming and structural choices. Under these conditions the contribution of optical and thermal solar panels is really appreciated. Table 1 shows the boundary conditions for growing tomatoes.

\subsection{Greenhouse Structural Description}

The greenhouse considered is an Artigianfer type STO construction with steel structure prefabricated. It is covered with glass cover horizontal beam pattern and small flaps with north-south orientation.

It has a width of $25.60 \mathrm{~m}$ divided into two spans of $12.80 \mathrm{~m}$. It is $150.107 \mathrm{~m}$ long and is divided into 39 sections ranging from $4.035 \mathrm{~m}$. The eaves height is $4.60 \mathrm{~m}$. In terms of structural elements, the greenhouse has cross doors.

Symmetric and transverse frames are stuck at the bottom and top. They are made with tubular columns $120 \times 80 \times 3 \mathrm{~mm}$ Fe 360 and horizontal beams lattice currents $80 \times 40 \times 3 \mathrm{~mm}$ 
Table 1: Climatic requirements of the tomato considered in this study.

\begin{tabular}{lcc}
\hline Minimum biological & ${ }^{\circ} \mathrm{C}$ & 6 \\
Maximum biological & ${ }^{\circ} \mathrm{C}$ & 30 \\
Thermal sums & ${ }^{\circ} \mathrm{C}$ & $1800-2000$ \\
Optimal substrate & ${ }^{\circ} \mathrm{C}$ & $15-20$ \\
Light intensity for maximum opening stomata & $\mathrm{Klux}$ & 10 \\
Minimum light intensity for starting photosynthesis & $\mathrm{Klux}$ & 2 \\
Maximum light intensity for starting photosynthesis & $\mathrm{Klux}$ & 20 \\
Minimum relative humidity & $\%$ & 56 \\
Maximum relative humidity & $\%$ & 92 \\
Daily optimal humidity & $\%$ & 67 \\
Nightly optimal humidity & $\%$ & 84 \\
\hline
\end{tabular}

Fe 430 tubular rods and rod wall. The roof rafters are made from the water canal collector and of pressed sheet metal. The side purlins are made of C-sections from $90 \times 50 \times 1,8 \mathrm{~mm}$ made of cool folded sheet. The glazing consists of rods 12 and $14 \mathrm{~mm}$ for roofs and walls. The calculation was performed in accordance with the requirements of the UNI-EN 13031-1 for greenhouses with metal structure. The maximum unit stress for steel Fe 360 of 1,600 kg/ $\mathrm{cm}^{2}$ for the first load cases and 1,800 kg $/ \mathrm{cm}^{2}$ for the other; for steel Fe 430 are of $1,900 \mathrm{~kg} / \mathrm{cm}^{2}$ for the first load cases and $2135 \mathrm{~kg} / \mathrm{cm}^{2}$ for the other. These have a corrosion protection due to the galvanizing bath.

The greenhouse consists of 8 very narrow aisles, each of $3.2 \mathrm{~m}$, characterized by two sloping roof pitches of $22^{\circ}$ degrees $(40 \%)$ and exposed north-south.

The greenhouse is equipped with continuous full-stop driven by motors with rack system if the temperature inside the greenhouse exceeds a given temperature. This automated system therefore depends on measurement of a temperature sensor located near the slopes. Outside the building is also home to a wind instrument, in the case of strong wind forces the system automatically recloses.

\subsection{Photovoltaic Greenhouse}

The structure of the PV greenhouse is the same as the glass greenhouse with the difference that on south-facing slopes are placed photovoltaic modules, glass is used wholly within the aquifer north. The photovoltaic greenhouse modeled was designed and built through cooperation between Artigianfer and Isofotòn. It consists of a $246.16 \mathrm{kWp}$ photovoltaic array that receives a fee of $0.43 € / \mathrm{kWh}$ for the full architectural integration instead of glass on flap south.

The system consists of 1456 high-efficiency modules Isofoton IS-170/24 transparent laminates, unframed, allowing full integration in place of windows. The distance between cells, studied in the design stage, allows the passage of light, making possible the operation of nursery underlying coverage. Under the cover are positioned 36 inverter SMA Sunny Mini Central $7000 \mathrm{TL}$, placed on metal structures to improve the visual impact.

The PV modules produced by Isofoton are made with pseudoquadrate monocrystalline silicon cells high efficiency for energy conversion of solar radiation into DC electricity.

The cell circuit is laminated using EVA (ethylene-vinyl acetate) as encapsulating a complex of tempered glass on the front and a plastic polymer (TEDLAR) on the back, 
Table 2: Details of a single photovoltaic module.

\begin{tabular}{ll}
\hline & Description \\
\hline Cell type & Monocrystalline, textured, antireflective layer \\
Dimensions & $125 \mathrm{~mm} \times 125 \mathrm{~mm}$ \\
Number of cells per module & 72 cells in series \\
& (1) Tempered glass and microstructured high transmissibility \\
Structure & (2) Cells laminated with EVA (ethylvinyl acetate) \\
& (3) Back-to-back tedlar/polyester layers \\
\hline
\end{tabular}

resistant to environmental agents and provided with electrical insulation. Details are shown in Table 2.

\subsection{Greenhouse Model}

Since TRNSYS 17 version three-dimensional geometry data created by Trnsys3d for TRNSYS can be imported into TRNBuild. Trnsys3d for TRNSYS is a plugin for Google SketchUp. This allows to create a building geometry from scratch: add zones, draw heat transfer surfaces, windows, shading surfaces, and so forth. The geometry data is divided into three groups.

(i) Building geometry.

(ii) External shading geometry.

(iii) GeoPosition geometry: This data is used to define the position for comfort calculation or radiative gains.

For all surfaces of the zone three-dimensional data is provided due to importing an IDF file. For this geometry mode, detailed internal and external radiation modes have been selected. Different radiation modes for direct and diffuse shortwave radiation and longwave radiation distribution are available within a thermal zone.

\subsubsection{Beam Radiation Distribution}

For external windows, shading and insolation matrices are used to distribute the primary solar direct radiation entering the zone. These matrices are based on the three dimensional data of the building and shader surfaces. This mode is used for simulating highly glazed zones where the correct distribution of direct solar radiation is important. For generating shading/insolation matrices TRNBuild calls an auxiliary tool based on TRNSHD. TRNSHD subdivides the celestial hemisphere into patches based on the so-called Tregenza model. The resolution of the sky division can be set to medium (577 patches) or high (2305 patches). For each center point of a patch the sunlit fraction of external windows with three-dimensional data is calculated and saved into the shading matrix file. In addition, a diffuse radiation sunlit factor is calculated assuming an isotropic sky and written into an *.SHM file. If no external window is shaded no file is generated. In addition to sunlit fractions of external windows, TRNSHD calculates the beam sunlit fractions of the window that strike each inside surface of the zone. These distribution factors for all patches are calculated and written into a separate file $\left({ }^{*}\right.$-xxx.ISM). 


\subsubsection{Diffuse Radiation Distribution}

For a detailed treatment of shortwave diffuse radiation including multireflection, the new radiation model applies so-called Gebhart factors. The key factor of this method is the view factor matrix. For generating the matrix TRNBuild calls an auxiliary program called TRNVFM. This mode is recommended for simulating highly glazed zones where diffuse radiation plays an important role.

\subsubsection{Longwave Radiation Exchange of Surfaces within a Zone}

For a detailed treatment of longwave radiation exchange including multireflection, the new radiation model applies so-called Gebhart factors. The key factor of this method is the view factor matrix. This mode is recommend for modeling the effect of low materials or detailed comfort analysis where the comfort depends on the location within the zone.

For calculating the view factor matrix TRNBuild calls an auxiliary tool called TRNVFM. For each zone with a detailed diffuse or longwave radiation mode the surface viewfactor matrix is calculated and written into a separate file (*VFM). In addition, the view factor vector for comfort and radiative gain positions are determined and saved to the same file.

\subsubsection{Direct Radiation}

For a detailed treatment of shortwave beam radiation shading and distribution the multizone building model reads in the sunlit factor matrices generated by TRNBuild at the beginning of the simulation. For each time step the current sunlit fraction of surfaces are determined by a bilinear interpolation of the four nearest center points with respect to the Sun's actual position. The matrices are used for distributing primary beam radiation entering a zone through external windows.

\subsubsection{Diffuse Radiation}

In order to use a mathematical description similar to the longwave case, all surfaces are assumed to be transparent. That means solar radiation enters the zone from outside. The surfaces are not emitting radiation. They are assumed to be "passive" because they are only reflecting, absorbing, and/or transmitting solar radiation originating from outside of the zone. Based on this idea again a (solar) Gebhart matrix can be created. For opaque surfaces (walls) the transmitted diffuse solar radiation is zero.

The derivation of the describing equations for the detailed diffuse solar radiation heat transfer is based on the following assumptions.

(1) All surfaces are assumed to be transparent (opaque surfaces are considered later).

(2) Radiation leaving a surface is indicated by a positive sign of the corresponding heat flux.

(3) $\rho_{\text {difsol }}$ is the hemispherical solar reflectivity.

The Gebhart-factor $G_{\mathrm{difsol}, j \rightarrow k}$ for diffuse solar radiation is defined as the fraction of transmitted solar radiation through surface $A_{j}$ that reaches $A_{k}$ surface and is not reflected. 
$G_{\text {difsol }, j \rightarrow k}$ includes all the paths for reaching $A_{k}$, that is, the direct paths and paths by means of one or multiple reflections. The abbreviation "difsol" stands for diffuse solar radiation.

Using the assumptions from above, the (dimensionless) Gebhart matrix for diffuse solar radiation can be written as follows:

$$
G_{\text {difsol }}=\left(I-F \rho_{\text {difsol }}\right)^{-1} F\left(I-\rho_{\text {difsol }}\right),
$$

where $\rho_{\text {difsol }}$ is a diagonal matrix. The variable $I$ describes the identity matrix, $F$ again stands for the view factor matrix.

Using the auxiliary matrix with dimension $G_{\mathrm{difsol}}^{*}\left[\mathrm{~m}_{2}\right]$ it can be shown that the net heat flux vector $\dot{Q}_{\text {difsol }}$ for diffuse solar radiation in an enclosure is given by

$$
\dot{Q}_{\text {difsol }}=G_{\text {difsol }}^{*} I_{\text {difsol }}
$$

with

$$
G_{\text {difsol }}^{*}=-G_{\text {difsol }}^{T} A
$$

$G_{\text {difsol }}^{T}$ is the transpose of $G_{\text {difsol }}$ and $A$ is the diagonal matrix describing the surface areas.

The driving force for diffuse solar radiation is the vector $I_{\text {difsol }}$ with one component $I_{\text {difsol, } k}$ for each surface $A_{k}$ of the enclosure. $I_{\text {difsol, } k}$ is equal to the transmitted part of the incident diffuse solar on the outside of surface $A_{k}$ leaving the inner side. Walls are perfectly opaque to solar radiation and therefore $I_{\mathrm{difsol}, k}^{\text {wall }}=0 . \dot{Q}_{\mathrm{difsol}}$ is determined by a matrix multiplication with the driving force $I_{\text {difsol }}$.

\subsubsection{Gebhart Method}

The detailed model for describing the heat exchange driven by longwave radiation exchange and convection. In comparison to the standard model there is no artificial star node, because the longwave radiative heat transfer is treated separately. The derivation of the describing equations for the detailed longwave radiation heat transfer is based on the following assumptions.

(1) Absorption of radiation on a surface is indicated by a negative sign of the corresponding heat flux whereas net emission means a positive heat flux.

(2) All surfaces are isothermal.

(3) All surfaces are perfect opaque for longwave radiation.

(4) All surfaces are (diffuse) gray. This signifies that emissivity and absorptivity do not depend neither on wavelength nor on direction.

(5) $\rho_{\text {ir }}$ is the hemispherical longwave reflectivity.

The Gebhart-factor $G_{i r, j \rightarrow k}[1,2]$ is defined as the fraction of the emission from surface $A_{j}$ that reaches surface $A_{k}$ and is absorbed. $G_{\mathrm{ir}, j \rightarrow k}$ include all the paths for reaching $A_{k}$, that is, the direct paths and paths by means of one or multiple reflections. The abbreviation IR stands for "infrared," meaning the longwave range of the radiation spectrum. 
Using the assumptions from above, the (dimensionless) Gebhart matrix for longwave radiation can be written as follows:

$$
G_{\text {ir }}=\left(I-F \rho_{\text {ir }}\right)^{-1} F \varepsilon_{\text {ir }}
$$

where $\rho_{\text {ir }}$ and $\varepsilon_{\text {ir }}$ are diagonal matrices describing reflectivity and emissivity, respectively. The variable $I$ describes the identity matrix. The view factor (written by $F$ ) is defined as the fraction of diffusely radiated energy leaving surface $A$ that is incident on surface $B$.

Introducing the auxiliary matrix $G_{\mathrm{ir}}^{*}$ with dimension $\left[W / T^{4}\right]$, it can be shown that the net heat flux vector $Q_{\mathrm{ir}}^{*}$ longwave radiation in an enclosure is given by

$$
\dot{Q}_{\mathrm{ir}}=G_{\mathrm{ir}}^{*} T^{4}
$$

with

$$
G_{\mathrm{ir}}^{*}=\left(I-G_{\mathrm{ir}}^{T}\right) A \varepsilon \sigma .
$$

$T$ is the temperature vector in the enclosure, $G_{\mathrm{ir}}^{T}$ is the transpose of $G_{\mathrm{ir}}, \sigma$ the StefanBoltzmann constant, and $A$ the diagonal matrix describing the surface areas. The auxiliary matrix $G_{\mathrm{ir}}^{*}$ only depends on optical (emissivity, reflectivity) and geometrical (view factor, area) properties as well as on the Stefan-Boltzmann constant. If this matrix is calculated first, the net heat flux of all surfaces in the enclosure can simultaneously be calculated by one simple matrix multiplication with the fourth power of the temperature vector.

\section{Results}

The project carried out with TRNSYS allows to extract all variables time-dependent, running simulations for hourly time periods established by the user, from a single hour to one year.

\subsection{Simulation of Solar Radiation}

Using the TRNSYS software has been run the simulation of the annual internal and external radiation in both types of structure and in the three selected locations. The results are shown in charts below (Figures 1, 2, and 3) where is possible to see the comparison between the annual internal and external solar radiation in both structures.

\subsection{Simulation of Energy Demand}

To compare energy consumption between PV greenhouse and glass greenhouse, the monthly energy consumptions were found for heating and cooling and compared to each other. These values were found for the three locations selected. The following tables (Tables 3, 4, and 5) show the monthly energy demand $(\mathrm{kWh})$ for heating and cooling necessary for the soil-less tomato. 


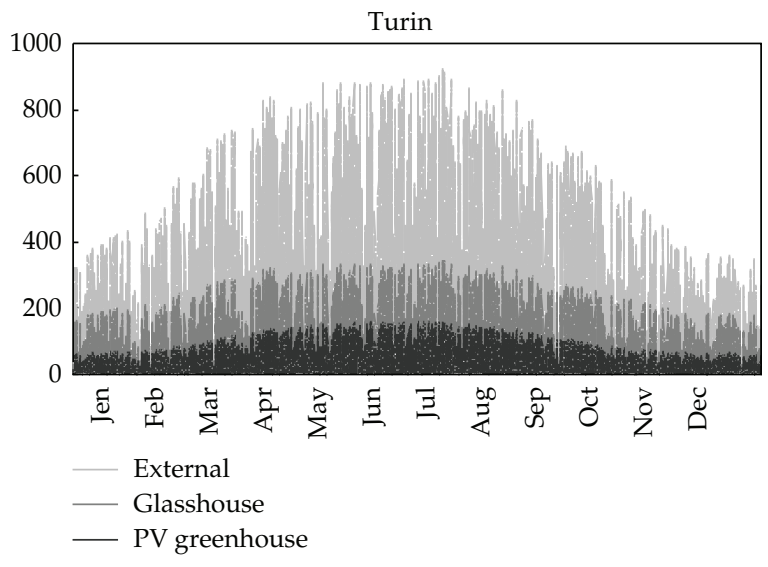

Figure 1: Hourly solar radiation $\left(\mathrm{W} / \mathrm{m}^{2}\right)$ on external and internal soil, annual simulation for both structures (glass and PV greenhouse) in Turin.

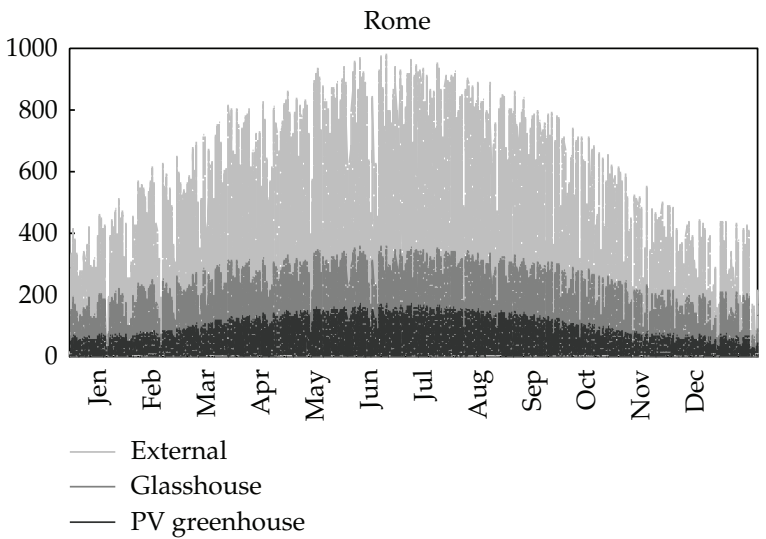

Figure 2: Hourly solar radiation $\left(\mathrm{W} / \mathrm{m}^{2}\right)$ on external and internal soil, annual simulation for both structures (glass and PV greenhouse) in Rome.

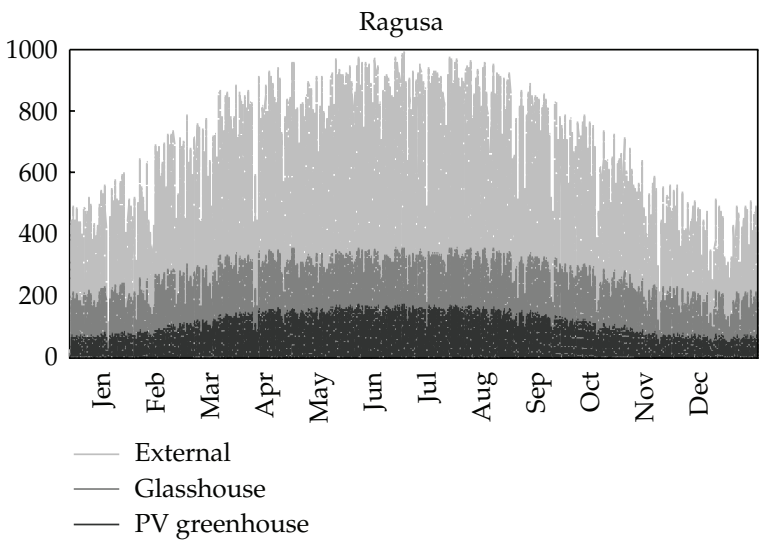

Figure 3: Hourly solar radiation $\left(\mathrm{W} / \mathrm{m}^{2}\right)$ on external and internal soil, annual simulation for both structures (glass and PV greenhouse) in Ragusa. 
Table 3: Monthly heating and cooling consumption $(\mathrm{kWh})$ for soil-less tomato cultivated in PV and glass greenhouse located in Turin, with the difference $(\mathrm{kWh})$ and the saving (variation percentage).

\begin{tabular}{lcccccccc}
\hline TURIN & \multicolumn{2}{c}{ Greenhouse } & \multicolumn{2}{c}{ PV greenhouse } & \multicolumn{2}{c}{ Difference } & \multicolumn{2}{c}{ Variation \% } \\
Month & Heating & Cooling & Heating & Cooling & Heating & Cooling & Heating & Cooling \\
\hline January & 53486 & 0 & 48518 & 0 & 4968 & 0 & 9.29 & 0.00 \\
February & 36776 & 0 & 32449 & 0 & 4327 & 0 & 11.77 & 0.00 \\
March & 11457 & 0 & 9725 & 0 & 1732 & 0 & 15.12 & 0.00 \\
April & 1658 & 0 & 850 & 0 & 807 & 0 & 48.70 & 0.00 \\
May & 0 & 20942 & 0 & 9810 & 0 & 11132 & 0.00 & 53.16 \\
June & 0 & 42804 & 0 & 27087 & 0 & 15717 & 0.00 & 36.72 \\
July & 0 & 60019 & 0 & 40326 & 0 & 19693 & 0.00 & 32.81 \\
August & 0 & 43565 & 0 & 25239 & 0 & 18326 & 0.00 & 42.07 \\
September & 0 & 16009 & 0 & 5149 & 0 & 10861 & 0.00 & 67.84 \\
October & 1276 & 0 & 939 & 0 & 337 & 0 & 26.40 & 0.00 \\
November & 21960 & 0 & 19382 & 0 & 2578 & 0 & 11.74 & 0.00 \\
December & 51478 & 0 & 45713 & 0 & 5764 & 0 & 11.20 & 0.00 \\
\hline
\end{tabular}

Table 4: Monthly heating and cooling consumption (kWh) for soil-less tomato cultivated in PV and glass greenhouse located in Rome, with the difference $(\mathrm{kWh})$ and the saving (variation percentage).

\begin{tabular}{lcccccccc}
\hline ROME & \multicolumn{2}{c}{ Greenhouse } & \multicolumn{2}{c}{ PV greenhouse } & \multicolumn{2}{c}{ Difference } & \multicolumn{2}{c}{ Variation } \\
Month & Heating & Cooling & Heating & Cooling & Heating & Cooling & Heating & Cooling\% \\
\hline January & 15774 & 0 & 13918 & 0 & 1856 & 0 & 11.77 & 0.00 \\
February & 8519 & 0 & 7755 & 0 & 765 & 0 & 8.98 & 0.00 \\
March & 2211 & 0 & 1723 & 0 & 488 & 0 & 22.06 & 0.00 \\
April & 179 & 13443 & 51 & 2904 & 128 & 10539 & 71.56 & 78.40 \\
May & 0 & 37213 & 0 & 20247 & 0 & 16966 & 0.00 & 45.59 \\
June & 0 & 58891 & 0 & 39235 & 0 & 19655 & 0.00 & 33.38 \\
July & 0 & 83357 & 0 & 57570 & 0 & 25787 & 0.00 & 30.94 \\
August & 0 & 70657 & 0 & 44271 & 0 & 26386 & 0.00 & 37.34 \\
September & 0 & 33263 & 0 & 14270 & 0 & 18993 & 0.00 & 57.10 \\
October & 14 & 8680 & 3 & 762 & 12 & 7918 & 81.17 & 91.22 \\
November & 3248 & 0 & 2581 & 0 & 667 & 0 & 20.53 & 0.00 \\
December & 11048 & 0 & 9891 & 0 & 1158 & 0 & 10.48 & 0.00 \\
\hline
\end{tabular}

\section{Discussion}

The new version of software, TRNSYS 17, together with Google SketchUp simplifies and reduces the time for building projects and simulation to improve the quality of the information structures in high-glass surface. Simulations show that for glasshouses, the increased level of detail has a high impact on the results but increases input effort and computing time.

The simulations show that the solar radiation inside the greenhouse is on average half that of the solar greenhouse glass. This could lead to a reduction in photosynthetic efficiency of plants. It could therefore be interesting to develop a model that divides the beam of sunlight into ododa possible to assess which wavelengths are present in the structures.

Simulations of heating and cooling energy demand show how different are the energy consumptions in three locations. The integrated photovoltaic roof saves energy for air 
Table 5: Monthly heating and cooling consumption $(\mathrm{kWh})$ for soil-less tomato cultivated in PV and glass greenhouse located in Ragusa, with the difference (kWh) and the saving (variation percentage).

\begin{tabular}{lcccccccc}
\hline RAGUSA & \multicolumn{2}{c}{ Greenhouse } & \multicolumn{2}{c}{ PV greenhouse } & \multicolumn{2}{c}{ Difference } & \multicolumn{2}{c}{ Variation \% } \\
Month & Heating & Cooling & Heating & Cooling & Heating & Cooling & Heating & Cooling \\
\hline January & 577 & 0 & 517 & 0 & 60 & 0 & 10.33 & 0.00 \\
February & 149 & 0 & 111 & 0 & 38 & 0 & 25.47 & 0.00 \\
March & 164 & 7372 & 93 & 730 & 71 & 6643 & 43.23 & 90.10 \\
April & 0 & 18560 & 0 & 4821 & 0 & 13739 & 0.00 & 74.02 \\
May & 0 & 41945 & 0 & 24305 & 0 & 17641 & 0.00 & 42.06 \\
June & 0 & 60700 & 0 & 41294 & 0 & 19406 & 0.00 & 31.97 \\
July & 0 & 78880 & 0 & 54848 & 0 & 24032 & 0.00 & 30.47 \\
August & 0 & 72675 & 0 & 46982 & 0 & 25693 & 0.00 & 35.35 \\
September & 0 & 48951 & 0 & 25404 & 0 & 23547 & 0.00 & 48.10 \\
October & 0 & 20986 & 0 & 5563 & 0 & 15423 & 0.00 & 73.49 \\
November & 61 & 0 & 44 & 0 & 17 & 0 & 28.29 & 0.00 \\
December & 306 & 0 & 251 & 0 & 55 & 0 & 17.87 & 0.00 \\
\hline
\end{tabular}

conditioning in all seasons: for all three locations considered it is on average of $30 \%$ for summer cooling and $11 \%$ for winter heating. In autumn and spring, these savings are much greater and in very different locations. This is due to temperatures close to those optimal for tomato and in some cases completely avoids the intervention of conditioning.

\section{References}

[1] M. Carlini, S. Castellucci, M. Guerrieri, and T. Honorati, "Stability and control for energy production parametric dependence," Mathematical Problems in Engineering, vol. 2010, Article ID 842380, 21 pages, 2010.

[2] M. Carlini, D. Monarca, P. Biondi, T. Honorati, and S. Castellucci, "A simulation model for the exploitation of geothermal energy for a greenhouse in the viterbo province," in Proceedings of the International Conference Ragusa SHWA, Work Safety and Risk Prevention in Agro-food and Forest Systems, pp. 621-629, Ragusa Ibla Campus, Italy, 2010.

[3] M. Carlini, D. Monarca, M. Cecchini et al., "Energetic and ergonomic aspects in the photovoltaic greenhouses," in Proceedings of the International Conference Ragusa SHWA, Work Safety and Risk Prevention in Agro-food and Forest Systems, pp. 576-629, Ragusa Ibla Campus, Italy, 2010. 


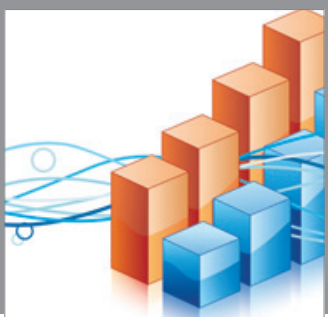

Advances in

Operations Research

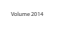

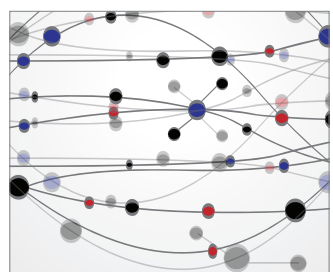

\section{The Scientific} World Journal
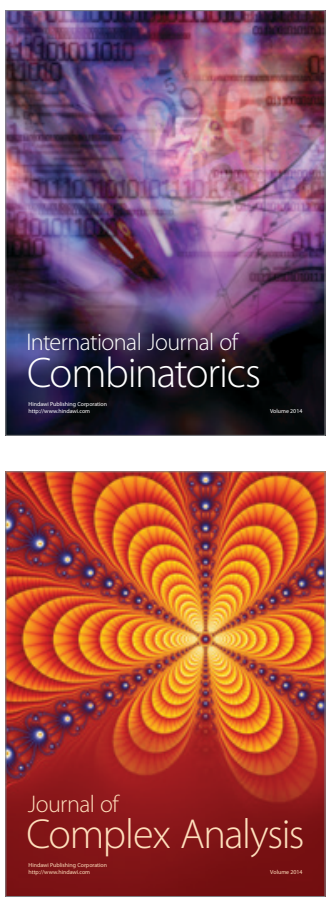

International Journal of

Mathematics and

Mathematical

Sciences
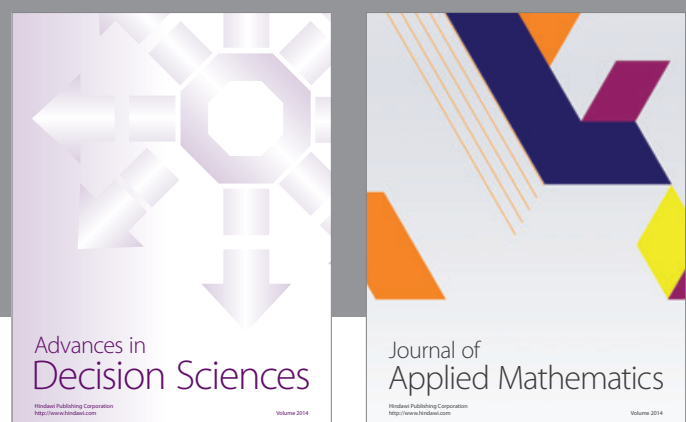

Journal of

Applied Mathematics
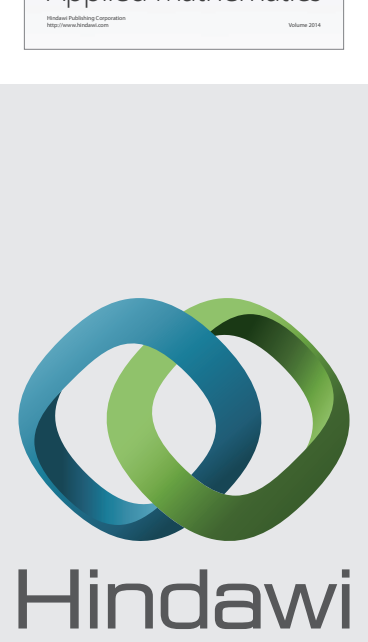

Submit your manuscripts at http://www.hindawi.com
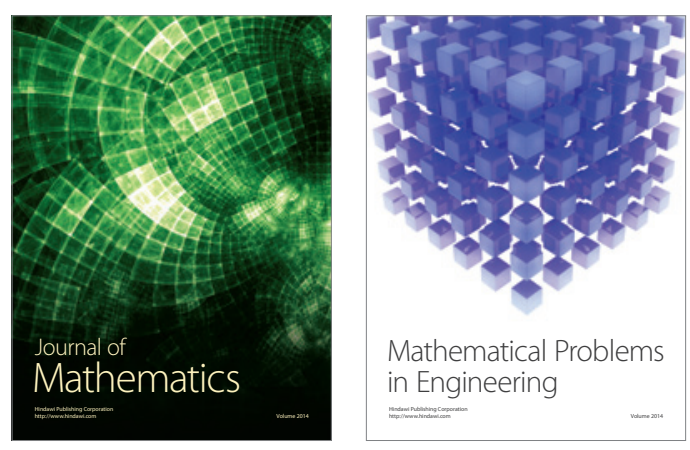

Mathematical Problems in Engineering
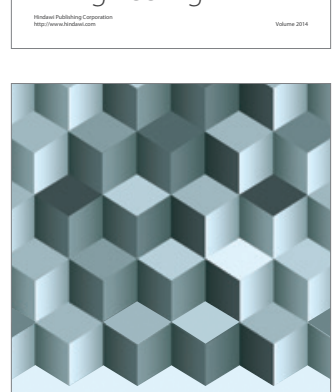

Journal of

Function Spaces
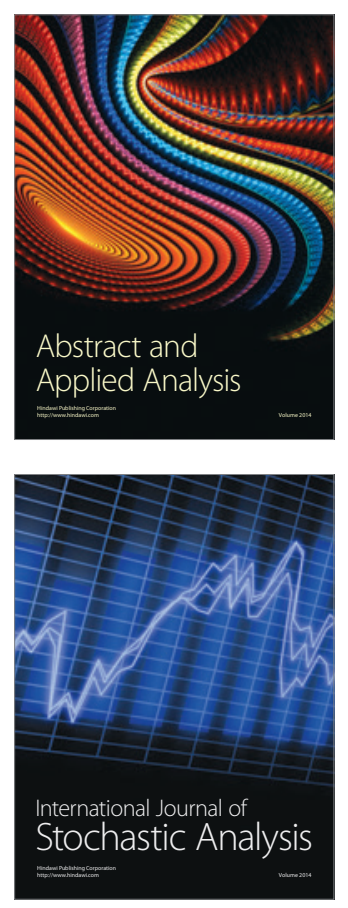

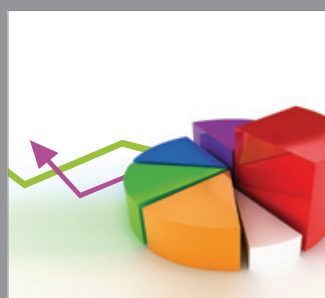

ournal of

Probability and Statistics

Promensencen
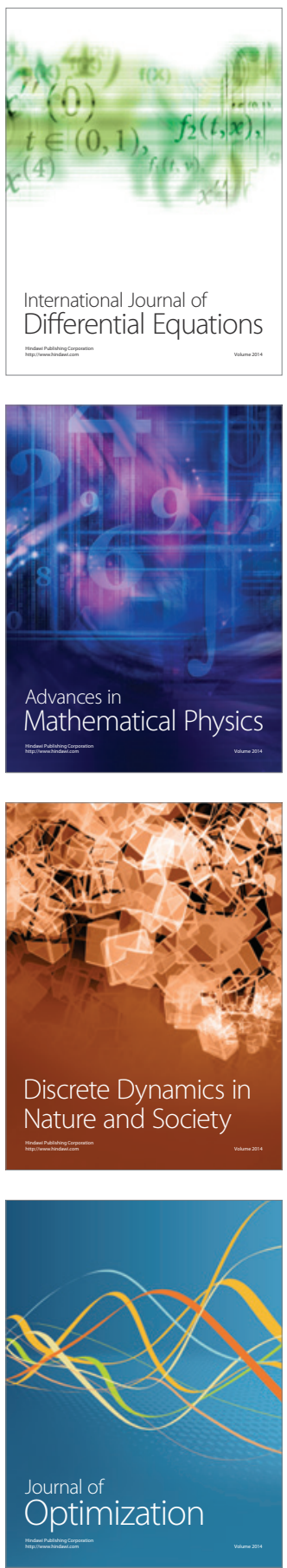